DOI: $10.3724 /$ SP.J.1218.2012.00764

\title{
A Navigation Method for Multiple Robots Based on a Single Mobile Node
}

\author{
JI Daxiong ${ }^{1}$, LIU Jian ${ }^{1,2}$ \\ (1. The State Key Laboratory of Robotics, Shenyang Institute of Automation, Chinese Academy of Sciences, Shenyang 110016, China; \\ 2. Graduate University of Chinese Academy of Sciences, Beijing 100049, China)
}

\begin{abstract}
To satisfy the requirements of independent navigation in low cost multiple robots, a navigation frame of masterslave robots with only one master robot is introduced briefly. A range-only navigation algorithm for slave robots of low cost is presented for the navigation in unknown current. This algorithm only needs a master robot. The master is just a single mobile node which has high navigation performance. Observability of this master-salve system is theoretically analyzed. The conditions of slave robot trajectory under which observability is guaranteed are evaluated by a slave robot in real-time. The slave takes appropriate action to control its heading to achieve observability if observability is lost on the current trajectory. The navigation algorithm is implemented by a standard extended Kalman filter, and it is able to acquire accurate estimation of robot's position. The simulation results show its effectiveness of the proposed algorithm.
\end{abstract}

Keywords: low cost; slave robot; range-only navigation; single mobile node; observability; heading control

\section{Introduction}

A new wave of robots has led to exciting scientific findings over the past decade. More and more mobile rather than stationary robots play an important role recently. Highly mobile exploration robots can reach places which are hard or impossible to access by humans. They provide a platform for their onboard sensors to collect data in an environment which has never been visited previously. The accuracy of position estimate is critical for robots, as unsupervised decisions are made based on the location estimates.

Multiple robots navigation is a key problem and is studied widely in the world. Vaganay ${ }^{[1]}$ studied the slave navigation method based on the ranges from two masters. The two masters own highly accurate navigation ability. The position estimates of slave ones are corrected by the accurate navigation information of the masters and ranges from masters. Baker ${ }^{[2]}$ proposed a method to determine the position of robot using not only ranges but also angles. In order to realize navigation for unlimited number of robots, Eustice ${ }^{[3]}$ introduced a time synchronous navigation mechanism. Master robot sends data to slave ones periodically. And the sending time is known by the slave ones. Then, every slave is able to obtain the range away from the master. The above method needs two masters for navigation. Scherbatyuk ${ }^{[4]}$ proposed a navigation method based on only one master named bottom beacon for underwater robots. A least mean square algorithm is adopted to estimate the robot position. And the trajectory of the robot should be bent other than straight. Gadre ${ }^{[5]}$ studied a low cost navigation method using extended Kalman filter with a single beacon. The observability of single beacon navigation system is proved in pole frame. Wei applied several robot fishes to gather pictures underwater with wireless navigation ${ }^{[6]}$. This method depends on extern wireless stations. $\mathrm{Xu}^{[7]}$ of Shenyang Institute of Automation of CAS established a digital simulation system to study navigation of multiple underwater robots deeply.

Most researches of range-only navigation algorithm concentrate on stationary node. Cooperative navigation based on a single mobile node has not been studied comprehensively. In this paper, the concept of cooperative navigation in which a group of mobile robots exchanges navigation information is proposed, and the challenges involved in implementing this concept are investigated and the solutions are proposed. Besides, the presented method only needs two distance measuring equipments of low cost. One of them is installed in the master mobile node, while the other is installed in the robot which only has navigation sensors of low cost. 


\section{System model}

To introduce the proposed method, it's applied to underwater robot navigation. There are two kinds of nodes in this method. One is a master robot with highly accurate navigation ability. The other is a slave robot with low cost sensors whose position estimate needs to be corrected. Each robot carries an acoustic modem for sending and receiving messages. The master sends message with position data of itself periodically. The slave knows the sending time previously. When the master's message is received by the slave, the range can be also obtained since the travel time of signal is measured. The slave corrects its navigation error with the information of master's position and ranges. As shown in Fig.1, $\xi O \eta$ is north-east-up coordinate. $x G y$ is ship coordinate where $G$ is the gravity center of slave robot. The slave sails in an horizontal plane carrying a cheap log and TCM2 electronic compass. $\psi$ denotes heading of the slave measured by electrical compass, $\gamma$ denotes heading of the trajectory, $\beta$ denotes drift angle, $\left(v_{\mathrm{E}}, v_{\mathrm{N}}\right)$ denote velocity bias in north-east-up coordinate between the measured and true values, $v^{\prime}$ denotes measured velocity of $\log$ along $x$ axis, and $\theta$ denotes pitch angle. The measured velocity $v$ in horizontal plane is given as below:

$$
v=v^{\prime} \cdot \cos \theta
$$

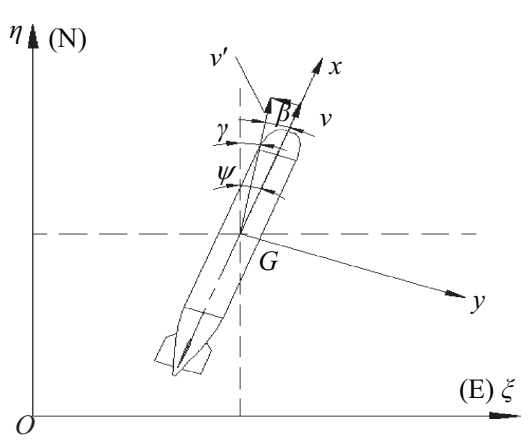

Fig.1 Motion of slave UUV (unmanned undersea vehicle) in current

The slave and the master, each carries a depth or altitude sensor which is used to acquire the vertical coordinate. Therefore the problem left is to obtain two horizontal coordinates of the slave in north-east-up coordinate. Unlike the navigation based on two or three nodes, it is impossible for slave to get its own position by one time of range measurement. Fortunately, it may be realized by the following principle: the master or slave carries out mobile tracking and gets as many independent measurements as possible at the same time, and the position of the slave can be estimated from the independent measurements. The principle depends on the observability of this navigation system. The input control of the slave or master should follow the principle.

Let $(\xi, \eta)$ denote the coordinates of the salve robot in north-east-up coordinate, $\left(v_{\mathrm{E}}, v_{\mathrm{N}}\right)$ and $\left(v_{\xi}, v_{\eta}\right)$ respectively denote the east and north component of velocity bias and robot velocity, $\left(\xi_{\mathrm{b}}(t), \eta_{\mathrm{b}}(t)\right)$ denote the position of master robot at time $t$. The position of master robot is received by the slave periodically. The nonlinear navigation system equation of the slave in simple form can be expressed as:

\section{State equation}

$$
\left[\begin{array}{c}
\dot{\xi}(t) \\
\dot{\eta}(t)
\end{array}\right]=\left[\begin{array}{c}
v_{\xi}(t)+v_{\mathrm{E}}(t) \\
v_{\eta}(t)+v_{\mathrm{N}}(t)
\end{array}\right]
$$

\section{Measurement equation}

$$
h(t)=\sqrt{\left(\xi(t)-\xi_{\mathrm{b}}(t)\right)^{2}+\left(\eta(t)-\eta_{\mathrm{b}}(t)\right)^{2}}
$$

\section{Navigation method}

\subsection{Observability}

Observability of a system is related to how the state affects the output. The position of the slave can be estimated if the system is observable. The method is based on the observability condition of slave robot navigation system.

Let

$$
\begin{aligned}
p & =\xi(t)-\xi_{\mathrm{b}}(t) \\
q & =\eta(t)-\eta_{\mathrm{b}}(t) \\
\boldsymbol{u} & =\left[\begin{array}{ll}
u_{\xi} & u_{\eta}
\end{array}\right]^{\mathrm{T}}=\left[\begin{array}{ll}
v_{\xi}+v_{\mathrm{E}} & v_{\eta}+v_{\mathrm{N}}
\end{array}\right]^{\mathrm{T}} \\
r & =h(t)=\sqrt{p^{2}+q^{2}}
\end{aligned}
$$

$s=s(t)$, which is the position of slave robot at time $t$.

Theorem 1 for observability is given as below:

Theorem 1(observability). The nonlinear systems (2) and (3) are observable at any time $t$, if $\left(u_{\xi} q-u_{\eta} p\right) \neq$ 0 .

Proof. 
The Lie derivative of $h$ with respect to $\boldsymbol{u}$ is:

$$
\begin{aligned}
\boldsymbol{L}_{u}^{1}(h) & =\frac{\partial h}{\partial s} \cdot \boldsymbol{u} \\
& =\left[\begin{array}{ll}
\frac{p}{r} & \frac{q}{r}
\end{array}\right]\left[\begin{array}{l}
u_{\xi} \\
u_{\eta}
\end{array}\right] \\
& =\frac{p u_{\xi}+q u_{\eta}}{r}
\end{aligned}
$$

By definition

$$
\boldsymbol{L}_{u}^{0}(h)=h(t)=r
$$

The gradients of $\boldsymbol{L}_{u}^{0}(h)$ and $\boldsymbol{L}_{u}^{1}(h)$ with respect to $s$ is

$$
\begin{aligned}
\frac{\partial \boldsymbol{L}_{u}^{0}(h)}{\partial s} & =\left[\begin{array}{ll}
\frac{p}{r} & \frac{q}{r}
\end{array}\right] \\
\frac{\partial \boldsymbol{L}_{u}^{1}(h)}{\partial s} & =\left[\begin{array}{ll}
\frac{u_{\xi} q^{2}-u_{\eta} p q}{r^{3}} & \frac{u_{\eta} p^{2}-u_{\xi} p q}{r^{3}}
\end{array}\right]
\end{aligned}
$$

Let

$$
\boldsymbol{O}=\left[\begin{array}{ll}
\frac{\partial \boldsymbol{L}_{u}^{0}(h)}{\partial s} & \frac{\partial \boldsymbol{L}_{u}^{1}(h)}{\partial s}
\end{array}\right]^{\mathrm{T}}
$$

Then

$$
\operatorname{det}(\boldsymbol{O})=\frac{1}{r^{2}}\left(u_{\xi} p-u_{\eta} q\right) \neq 0
$$

Thus

$$
\operatorname{rank}\left(\boldsymbol{O}\left(t_{0}\right)\right)=2
$$

The nonlinear systems (2) and (3) are observable ${ }^{[8]}$.

\subsection{Navigation algorithm}

The heading $\psi$ should be controlled in order to satisfy the observability condition which is expressed as

$$
\left(u_{\xi} q-u_{\eta} p\right) \neq 0
$$

That is

$$
\left(v_{\xi}+v_{\mathrm{E}}\right) q-\left(v_{\eta}+v_{\mathrm{N}}\right) p \neq 0
$$

where

$$
v_{\xi}=v \sin \psi \quad \text { and } \quad v_{\eta}=v \cos \psi
$$

In order to obtain the solution of inequation (5), we change the inequation into the following equation

$$
q \sin \psi-p \cos \psi=-q \frac{v_{\mathrm{E}}}{v}+p \frac{v_{\mathrm{N}}}{v}
$$

And it is obvious that

$$
\sin ^{2} \psi+\cos ^{2} \psi=1
$$

The opposite solution of inequation (5) can be deduced from equations (6) and (7)

$$
\cos \psi=\frac{k_{1} p \pm k_{2}|q|}{p^{2}+q^{2}}
$$

where

$$
k_{1}=-\left(p \frac{v_{\mathrm{N}}}{v}-q \frac{v_{\mathrm{E}}}{v}\right) \text { and } k_{2}=\sqrt{p^{2}+q^{2}-k_{1}^{2}}
$$

The $\psi$ which satisfies equation(8) is the heading of unobservability.

Thus controlled variable $\psi$ is obtained as below:

$$
\psi \neq \cos ^{-1} \frac{k_{1} p \pm k_{2}|q|}{p^{2}+q^{2}}
$$

The $\psi$ which satisfies inequation (9) is the heading of observability.

Because the velocity bias is not known previously, it is difficult to find trajectories which do not satisfy the observability condition in inequation (4). But we can show that simple trajectories are satisfied, such as a fold line trajectory. A standard extended Kalman filter (EKF) is adopted to estimate the position and velocity bias along the slave trajectory. Then the heading of slave robot is controlled by inequation (9) using the estimate of velocity bias in real time to avoid loss of observability on the current trajectory. The range-only navigation algorithm needs to be initialized with an absolute position $s_{0}$ from an appropriate sensor. Usually this is a GPS receiver.

The navigation algorithm is described as below:

\section{1. repeat}

2. wait

3. until $s(t)=s_{0}$

4. slave robot moves in a fold line with $\psi(t)$

5 . loop $\{$ for each sample period $\mathrm{d} t\}$

6. $\boldsymbol{u}=\left[\begin{array}{c}v \cos \psi(t-1)+v_{\mathrm{E}}(t-1) \\ v \sin \psi(t-1)+v_{\mathrm{N}}(t-1)\end{array}\right]$

7. $s(t)=s(t-1)+\boldsymbol{u} \cdot \mathrm{d} t$

8. if message $\boldsymbol{m}(t)$ is received from the master, then

9. $\boldsymbol{m}(t)=\left[\begin{array}{c}\xi_{\mathrm{b}}(t) \\ \eta_{\mathrm{b}}(t) \\ d_{\mathrm{b}}(t)\end{array}\right]$ where $d_{\mathrm{b}}(t)$ is the depth of master

10. $r(t)=h(t)=\sqrt{R^{2}-\left(d-d_{\mathrm{b}}\right)^{2}}$ where $R$ is the measured range between the master and the slave, and $d$ is the depth of slave

11. use EKF to do estimation, $s(t) \rightarrow \tilde{s}(t)$

12. end if

13. if estimates $\left(v_{\mathrm{E}}, v_{\mathrm{N}}\right)$ is available, then

14. $k_{1}=-\left(p \frac{v_{\mathrm{N}}}{v}-q \frac{v_{\mathrm{E}}}{v}\right)$

15. $k_{2}=\sqrt{p^{2}+q^{2}-k_{1}^{2}}$ 
16. control $\psi(t) \neq \cos ^{-1} \frac{k_{1} p \pm k_{2}|q|}{p^{2}+q^{2}}$

17. end if

\section{8. end loop}

\section{Simulation experiments}

Assuming that error variance of range measurements is $1 \mathrm{~m}$, velocity bias is $(0.5 \mathrm{~m} / \mathrm{s}, 0.5 \mathrm{~m} / \mathrm{s})$, the update period of navigation sensor data is $1 \mathrm{~s}$, range update period is $10 \mathrm{~s}$, and the velocity of the master and slave is $1.5 \mathrm{~m} / \mathrm{s}$. The master moves in a straight line, while the slave moves in a fold line. Trajectories of the master and slave are shown in Fig.2. The slave uses a GPS receiver to get its absolute position when it is on the surface. The GPS receiver on the slave has a circular error about $10 \mathrm{~m}$. During a flight, the slave uses the last known GPS position as the initial position estimate for the range navigation algorithm. These positions are converted into the Earth-fixed coordinate frame. In this particular movement, the slave heading commands are $150^{\circ}, 195^{\circ}, 150^{\circ}$ and $120^{\circ}$. The master heading command is $157.5^{\circ}$. Ranges from the master are measured by the range modem. The modem is set up to ping the master every $10 \mathrm{~s}$.

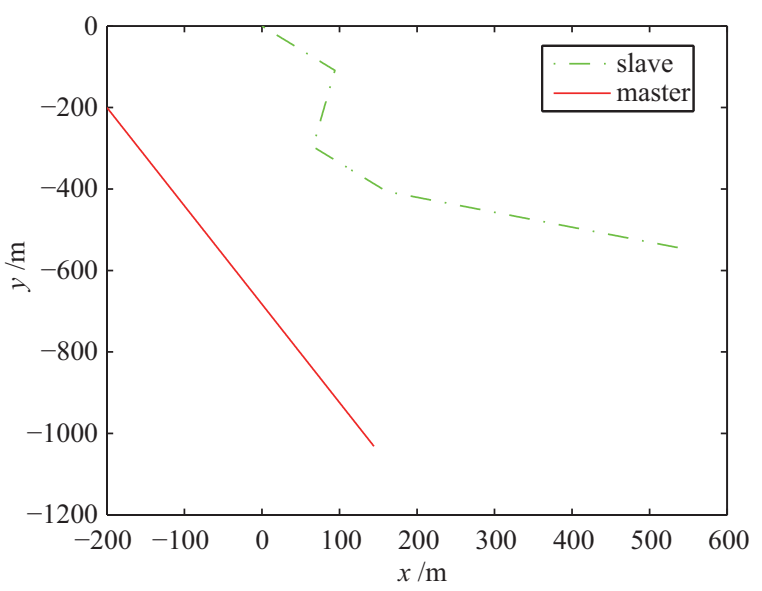

Fig.2 Trajectories of the master and slave

Fig. 3 shows the slave trajectory estimated by the range-only navigation algorithm of EKF. At the start of the movement, the slave position estimate computed by EKF is significantly away from the true solution. However as the EKF converges, the trajectory estimated keeps close to the true solution. Fig.4 shows the currents estimated by the range-only navigation algorithm. The initial change in the EKF estimates of currents is due to the GPS error of the initial position estimate. When a valid range measurement is available, the EKF adjusts the currents estimate to compensate for the GPS error. As more range measurements become available, estimated currents converge to true solution. Then the heading of slave robot for guaranteeing observability is obtained in real time from (9). The slave controls its heading for avoiding unobservability. At first, the slave starts a mobile trajectory and changes its heading for current estimation. The time of this simulation lasts about $350 \mathrm{~s}$. When the current has been estimated at the end of the mobile trajectory, the heading of unobservability is calculated by the slave in real time, as show in Fig.5. Then the slave controls its heading to avoid the unobservable heading for accurate navigation. The heading is controlled between the two unobservable headings. In this simulation, the heading is controlled with $120^{\circ}$ which is unequal to any of the unobservable headings in the simulation.

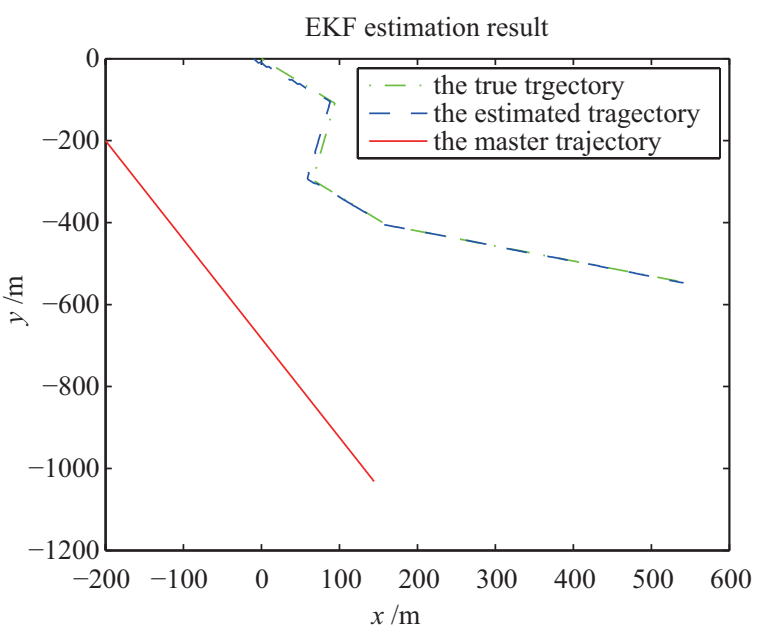

Fig.3 Simulation of slave trajectory estimated
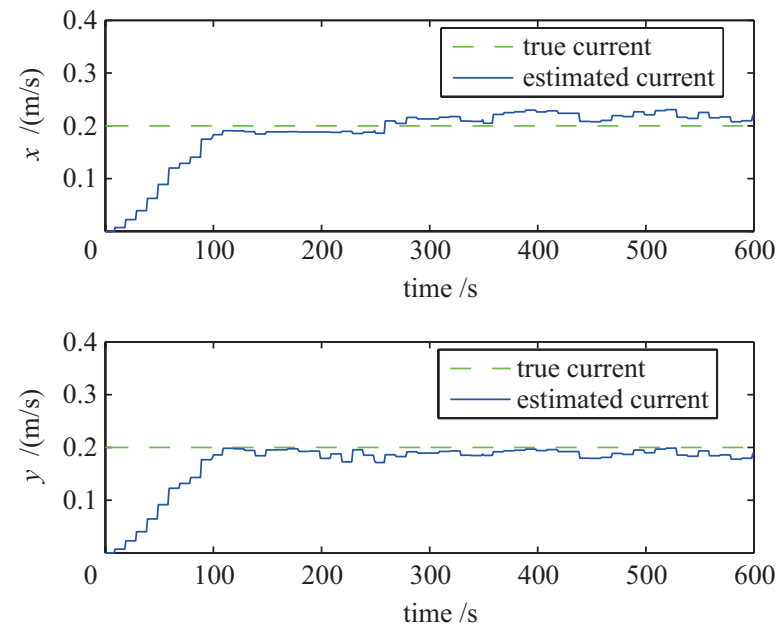

Fig.4 Simulation of current estimation 


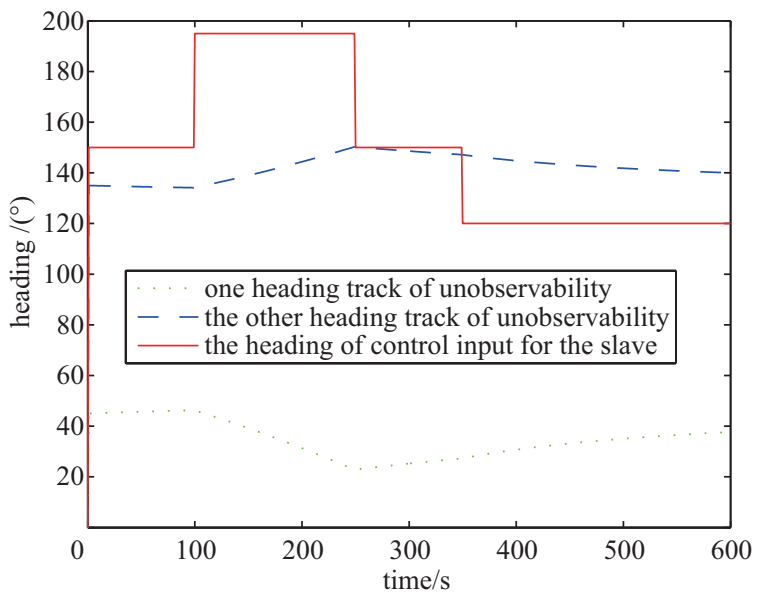

Fig.5 Headings for unobservability and observability

\section{Conclusions}

A range-only navigation method based on a single mobile robot has been proposed that is suitable for low cost robots. The principal contribution of this work is the precise observability analysis that characterizes observable robot trajectories in the presence of unknown velocity bias. Based on the observability condition, the controlled heading is deduced for slave robot. The conditions of slave robot trajectory under which observability is guaranteed can be evaluated by a slave robot in real-time and the slave can take appropriate action to change its heading to achieve observability if it determines that it is moving on an unobservable trajectory. The navigation algorithm is implemented by a standard extended Kalman filter. The results of simulation experiment show its validity. This method can be applied to multiple robots.

\section{References}

[1] Vaganay J, Leonard J J, Curcio J A, et al. Experimental validation of the moving long base-line navigation concept[C]// IEEE/OES Autonomous Underwater Vehicles. Piscataway, NJ, USA: IEEE, 2004: 59-65.

[2] Baker B N, Odell D L, Anderson M J, et al. A new procedure for simultaneous navigation of multiple AUV's[C]//OCEANS. Piscataway, NJ, USA: IEEE, 2005: 1-4.

[3] Eustice R M, Whitcomb L L, Singh H, et al. Experimental results in synchronous-clock one-way-travel-time acoustic navigation for autonomous underwater vehicles[C]//IEEE International Conference on Robotics and Automation. Piscataway, NJ, USA: IEEE, 2007: 4257-4264.

[4] Scherbatyuk A P. The AUV positioning using ranges from one transponder LBL[C]//OCEANS. Piscataway, NJ, USA: IEEE, 1995: 1620-1623.

[5] Gadre A S, Stilwell D J. A complete solution to underwater navigation in the presence of unknown currents based on range measurements from a single location[C]//IEEE/RSJ International Conference on Intelligent Robots and Systems. Piscataway, NJ, USA: IEEE, 2005: 1420-1425.

[6] Wei H X, Wang T M, Liang J H, et al. Control and maneuverability of multi-robofish[J]. Shipbuilding of China, 2003, 44(4): 80-85.

[7] $\mathrm{Xu} \mathrm{H} \mathrm{L,} \mathrm{Xu} \mathrm{Z} \mathrm{Z,} \mathrm{Feng} \mathrm{X} \mathrm{S.} \mathrm{Design} \mathrm{and} \mathrm{implementation}$ of a multiple underwater vehicle simulation system based on LAN[J]. Robot, 2005, 27(5): 423-425.

[8] Hermann R, Kerener A. Nonlinear controllability and observability[J]. IEEE Transactions on Automatic Control, 1977, 22(5): 728-740.

\section{About Authors:}

JI Daxiong (1981 -), male, Ph.D., Associate researcher. Research interests include Navigation and positioning of AUV, AUV control.

LIU Jian (1962-), male, Mater, Researcher. Research interests include general technology of AUV, AUV control. 